\title{
L'ACTE DE JUGER EN JE(U) : KAFKA, ARAGON, DERRIDA
}

\section{Charlotte Dufour}

BSN Press | « A contrario »

2020/1 n $30 \mid$ pages 111 à 135

ISSN $1660-7880$

Article disponible en ligne à l'adresse :

https://www.cairn.info/revue-a-contrario-2020-1-page-111.htm

Distribution électronique Cairn.info pour BSN Press.

(C) BSN Press. Tous droits réservés pour tous pays.

La reproduction ou représentation de cet article, notamment par photocopie, n'est autorisée que dans les limites des conditions générales d'utilisation du site ou, le cas échéant, des conditions générales de la licence souscrite par votre établissement. Toute autre reproduction ou représentation, en tout ou partie, sous quelque forme et de quelque manière que ce soit, est interdite sauf accord préalable et écrit de l'éditeur, en dehors des cas prévus par la législation en vigueur en France. Il est précisé que son stockage dans une base de données est également interdit. 


\title{
L'acte de juger en je(u): Kafka, Aragon, Derrida
}

\author{
Charlotte Dufour
}

\section{Un droit de travers}

«Juger, disons-nous d'abord, c'est trancher» (Ricœur 1992: 20), écrit Paul Ricœur dans L'acte de juger. D'un côté, un jeune garçon enamouré qui décide d'emprunter à son ami quelques ducats pour rendre visite à sa dulcinée; de l'autre, un créancier intransigeant dont les prétentions sont rigoureusement stipulées dans le contrat. Se mêlent encore à la situation de fait une tempête malheureuse, des retards de paiement et un vieux rêve de vengeance ${ }^{1}$. Dans une configuration binaire, le cas oppose alors deux parties et le juge somme toute tranche "en vue de mettre un terme à l'incertitude" (ibid.). Le geste traduit à cet égard, comme le rappelle notamment François Ost, « la fonction canonique du juge-arbitre, dans le modèle légaliste-libéral ", puisqu'il est traditionnellement exigé de ce dernier qu'il parachève " des contestations portant sur des droits et réalis[e] ainsi, dans l'espèce litigieuse, la volonté de la loi " (Ost 2012: 21). Cette "finalité courte» du procès exprime non seulement la nécessité à la fois sociale, juridique et psychologique de mettre un terme à l'attente en clôturant la procédure, mais également " la force du droit; bien plus, [elle] dit le droit dans une situation singulière» (Riccur 1992 : 20, 22).

Dire le droit comporte cependant une zone d'ombre, car avant de trancher, le juge doit d'abord décider. Dépourvu de formules magiques - le fameux précepte appliquer la loi étant singulièrement trompeur -, il procède à tâtons et entreprend de fait un véritable «saut dans le vide» (Ost 2015: 8), rompant avec les idées reçues d'une justice raisonnable. "Est-ce [toutefois] choquant que le droit agisse dans une nuit d'ignorance? " interroge Rainer Maria Kiesow; non, "car si on savait, un jugement ne serait justement plus nécessaire, il suffirait de trouver la solution" (Kiesow 2014b: 207). Autrement dit, une décision juridique ne constitue pas un simple résultat, dès lors qu'elle ne se déduit pas d'une seule opération mathématique. Les exemples en ce sens abondent: un même cas peut conduire à des conclusions différentes; un même juge

1 Le cas emprunte au célèbre procès du Marchand de Venise (1598) de William Shakespeare. 
peut quant à lui trancher en faveur d'arrêts opposés. Kiesow conçoit à ce titre l'image d'un « même [qui] se balade, s'égare, se perd dans les halls" (Kiesow 2014a: 11) des tribunaux, un même sans cesse menacé par la jurisprudence. Aléatoire, imprévisible, instable, "voilà une vision du droit qui vaut la peine d'être réfléchie» (ibid.: 12). Parce que «l'acte de juger a le volet conventionnel d'appliquer les règles de droit mais aussi de les négocier» (Bidima 2002: 200, je souligne), celui-ci révèle un espace qui court, "qui ne tient pas en place, [un] droit qui se déploie en possibilités" (Kiesow 2014a: 11) et au sein duquel la loi ne peut s'appréhender que dans la «dérobade perpétuelle» (Bidima 2002: 200). Et c'est bien parce que la décision est une entreprise aussi périlleuse qu'elle est confiée à des magistrats impartiaux. Toutefois, le système juridique est une tête de Janus au double visage (Jhering 2006:3), car derrière la neutralité des juges imposée par les Codes $^{2}$ se dévoile la face cachée: le juge, à défaut d'être une machine, est un être humain doué d'une sensibilité et d'une personnalité, dont la subjectivité peut jouir d'une certaine puissance.

"Le droit est le plus froid des monstres froids" (Kiesow 2014a: 9o); la maxime se prête parfaitement à deux récits de la première moitié $\mathrm{du} \mathrm{XX}^{\mathrm{e}}$ siècle - La colonie pénitentiaire (In der Strafkolonie) de Franz Kafka et Le droit romain n'est plus de Louis Aragon -, car la cause du tourment, en l'espèce, n'est pas seulement la loi, elle est d'abord ce que les juges en font. Le premier, écrit en 1914 et publié en 1919, est l'un des rares textes édités du vivant de l'auteur. L'action se situe dans un lieu inconnu et lointain (Bareït 2016: 255), où un officier présente à un voyageur la machine devant servir à la condamnation à mort prochaine de l'un de leurs prisonniers, plaçant ainsi au cœur de la nouvelle le système judiciaire et ses déviances. Au fil des explications, le visiteur se voit confronté à l'absurdité de la procédure, dans la mesure où le condamné n’a non seulement pas été informé des raisons de son exécution, mais il n’a pu en outre se défendre devant un tribunal. La réponse de l'officier est sans appel: il est inutile de donner au condamné des explications puisqu'il les apprendra sur son corps, révélant le supplice déclenché par la herse de la machine. Le voyageur, choqué, annonce qu’il se rallie à l'opinion majoritaire pour l'abolition de la pratique, un dénouement que ne peut supporter l'officier, si fier de cette invention, l'amenant alors, par loyauté, à faire danser les aiguilles sur sa propre chair. Au même moment, la machine s'affaire et se démantèle, se retournant contre son partisan dont le cadavre termine embroché sur les dents de l'appareil.

\footnotetext{
2 Pour exemple: article 6 de la Convention européenne des droits de l'homme (CEDH); article 29 alinéa 1 de la Constitution fédérale.
} 
Le second texte, écrit «dans la colère d'un temps où les faits parlaient plus haut que le sens humain" (Aragon 2000: 1253), constitue le septième et dernier conte du recueil Servitude et grandeur des Français $(1945)^{3}$, composé de récits publiés de manière partiellement clandestine ${ }^{4}$ durant la Résistance. Le droit romain n'est plus traduit les violences de l'été 1944 dont Aragon a été témoin et appelle à restaurer sans plus attendre les institutions juridiques (Bougnoux 2008: 203). Le droit romain ayant été aboli au profit du droit nazi, le Commandant von Lüttwitz-Randau, juge militaire, proclame sans compter et sans véritable procès de nombreuses condamnations à mort, le seul événement intéressant aux yeux de sa secrétaire, Lotte, qui s'ennuie éperdument. Lors d'une escapade à la campagne, durant laquelle ils s'égarent à la suite d'une erreur de train, Lotte et le Commandant, "se croyant encore en situation d'aller en partie fine" (Aragon 2010: 137), se font arrêter par un groupe de maquisards. Si les Français donnent l'occasion au juge de se défendre durant un procès un peu particulier - en parcourant les villages détruits par les idéologies hitlériennes et en s'arrêtant notamment devant une grande ferme abandonnée sur la porte de laquelle un enfant âgé de six ans a été cloué devant sa mère -, ce dernier se prend dans les mailles du filet de ses propres convictions, revendiquant avec ferveur la fidélité et l'amitié qu'il voue au Führer. Le Commandant est alors exécuté le lendemain matin, à quelques pas du lieu emblématique où sept personnes ont été tuées, dont une femme avec son enfant de dix-sept mois et un célèbre docteur ${ }^{5}$.

Les deux textes opèrent par conséquent un changement de paradigme intéressant, car ils font le jugement des juges, ou des exécuteurs - la distinction n'opérant plus ici. Chez Aragon, le juge militaire est en effet condamné par ses victimes alors que le dispositif judiciaire chez Kafka fait l'objet d'une accusation à la fois juridique et morale par l'expérimentation sanglante de son bien-fondé. Outre ces scènes d'exécution, les récits partagent encore une modalité du discours singulière, dès lors que la voix est donnée presque exclusivement aux bourreaux. La parole des juges, dans les deux cas,

\footnotetext{
3 Dans le numéro du 5 septembre 1944 de La Drôme en armes, journal fondé par Elsa Triolet et Louis Aragon durant les derniers mois de l'Occupation, Aragon fait paraître, sous le pseudonyme Georges Meyzargues, un article intitulé «Épilogue d'un drame », l'histoire réelle du Commandant von Lüttwitz-Randau qui a servi à l'élaboration de la nouvelle. L'article est en outre suivi d'un petit texte relatif à l'assassinat du docteur Bourdongle, dont la fin tragique est également relatée dans le récit.

4 C'est le cas de «Les bons voisins ", «Pénitent 1943» et «Le mouton».

5 Le docteur en question est le docteur Bourdongle, précédemment mentionné, dont l'histoire est relatée par André Viollis dans le numéro du 5 septembre 1944 de La Drôme en armes. Le matin du 19 mars 1944, deux officiers de la Gestapo frappent à la maison du docteur, car ce dernier a soigné des maquisards. Refusant d'entrer dans le rang, il est battu à mort. Dans son article «Épilogue d'un drame», Aragon précise quant à lui : «de tous les martyrs de la Drôme, le Docteur Bourdongle, de Nyons, restera l'un de ceux dont le souvenir sera le plus vivant" (Aragon 2010: 137).
} 
institue un rapport au langage et au corps symptomatique de leur puissance. Une puissance d'autant plus troublante qu'elle ne revendique à aucun moment la neutralité du système pénal, les deux figures d’autorité - l'officier et le Commandant n’appelant qu'à leur idéologie propre. Cet aspect du droit, troublant, gagne à cet égard à être déployé - déplié dirait Derrida ${ }^{6}$ - par la forme littéraire, car elle a l'avantage de révéler les strates du réel afin d'en dégager les contours les plus subtils, les plus insaisissables aussi, abandonnant la théorie pour la vie et ses aléas.

Derrida, justement. Son apport à la philosophie du droit est parfois sous-estimé, 114 ses réflexions n'étant pas centrées sur les questions juridiques. Cependant, sa contribution oblique, pour reprendre ses mots (Derrida 2005: 26), est significative en théorie $\mathrm{du}$ droit du point de vue du concept de la déconstruction notamment, puisqu'il permet de penser l'instabilité de certains binômes à la fois contradictoires et indissociables, tels que droit naturel/droit positif, droit matériel/droit formel, loi/droit ou encore droit/justice. Si le théoricien entreprend ces réflexions dans plusieurs de ses textes7, c'est dans Force de loi. Le «Fondement mystique de l'autorité» (1994) que sa pensée est la plus vive. Issu d'un discours (" Du droit à la justice») prononcé en octobre 1989 dans le cadre d'un colloque ${ }^{8}$, le texte présente trois apories constitutives de l'acte de juger: l'épochè de la règle, l’indécidable et l'urgence à juger. La seconde mérite, à la lumière des deux nouvelles, une attention toute particulière. Selon Derrida en effet, une décision judiciaire ne peut être juste que si elle fait au préalable l'épreuve de l'indécidable, une expérience impossible mais indispensable à tout jugement, l'hypothèse ébranlant alors sérieusement la notion de certitude, dès lors qu'elle en déconstruit la présomption.

Le but de cet article est ainsi de déstabiliser la conviction d'un droit profondément bienveillant et de rendre compte des limites de ce dernier lors de son applicabilité d'une part et de sa relation étroite avec la notion de pouvoir d'autre part. Sans pour autant «considérer la littérature comme un simple réservoir d’exemples ", il s'agira

\footnotetext{
6 Pour reprendre l'un de ses titres: Déplier Ponge.

7 Du droit à la philosophie (1990), Donner la mort (1992), Le siècle et le pardon (2001) et La bête et le souverain (2008) notamment.

8 Le colloque, intitulé «La déconstruction et la possibilité de la justice» (Deconstruction and the Possibility of Justice), s'est tenu à New York à la Cardozo Law School. Seule la première partie du texte (" Du droit à la justice») est lue. La seconde, "Prénom de Benjamin", n’est pas prononcée, mais elle est distribuée aux participants. Elle sera toutefois proclamée l'année suivante (1990) lors du colloque organisé à l'Université de Californie à Los Angeles ("Nazism and the "Final Solution": Probing the Limits of Representation»). Si « Du droit à la justice» traite de la question du jugement, «Prénom de Benjamin » s’intéresse davantage au lien entre droit et violence.
} 
plutôt de "se laisser [...] décentrer et interpeller par [son] imaginaire propre» (Axt 2017: 251), à l'occasion d'une réflexion portant sur l'acte - et l'art - de juger. Pour ce faire, un cheminement à rebours sera privilégié au travers d'une lecture croisée (Kafka, Aragon, Derrida). Une première partie se concentrera sur l'exécution du jugement lui-même, en interrogeant la signification du mot «machine» employé dans les deux textes de fiction, témoin et réceptacle de la machine judiciaire, et de son lien intrinsèque à la violence. Un intérêt spécifique sera accordé au parallélisme entre corps et pouvoir, afin de mettre en évidence les liaisons (dangereuses) qu'ils entretiennent. Lacte de juger à proprement parler fera quant à lui l'objet d'une seconde partie, dans le dessein de réfléchir à ses implications, bien plus complexes qu'une seule application stricte de la loi, un constat invitant notamment à distinguer la loi du droit. Enfin, une dernière partie approfondira un aspect inhérent, en amont, à chaque jugement: l’individualité du juge.

\section{Exécuter}

La machine judiciaire

«Il sentait le cercle menaçant se resserrer autour de lui, et le doigt pris dans l'engrenage redoutable de la machine judiciaire, il comprenait qu'il lui faudrait faire un gigantesque effort pour ne pas y laisser le bras tout entier " (Level 1908: 256, je souligne), écrit le narrateur de L'épouvante de Maurice Level à propos d'Onésime Coche, alors acculé sur le banc des accusés. La machine. Mise en branle une fois la procédure judiciaire enclenchée, elle constitue de fait un topos littéraire à même de signifier les rouages redoutables de la bête humaine ${ }^{9}$ du tribunal, ses réquisitoires, ses jugements et ses désarrois. Ce n'est à cet égard pas un hasard si le terme est répété dans les récits de Kafka et d'Aragon pour caractériser le système juridique défendu par les protagonistes, le Commandant (Le droit romain n'est plus) et l'officier (La colonie pénitentiaire). «Ce que j'appelle le droit germanique, jus germanicum ", proclame le juge militaire allemand von LüttwitzRandau, c'est «d'avoir à sa disposition une machine de guerre contre l'ennemi » (Aragon 2000 : 1225, je souligne). Autrement dit, le Commandant opère une (con)fusion et fait du droit nazi un instrument armé dont le dessein est de «justifier la victoire " (ibid.). Il en précise rapidement les contours, en insistant sur l'avant-gardisme d'une telle machination : « Notre Führer, en matière de droit, est, il faut dire, tout à fait inspiré. La suppression de toutes les lois au bénéfice de l'intérêt national [...], c'est une audace vraiment allemande." (Ibid.) Le dispositif juridique revendiqué consiste ainsi à abolir le droit romain, à savoir « la loi écrite, codifiée, la loi mise en paragraphes qui constitue

9 En référence au roman d'Émile Zola, La bête humaine (189o). 
le fondement d'un État de droit, d'un État démocratique " (Babilas 2004: 212), au profit d'un droit dépourvu de toute codification.

L'institution d'un tel dispositif juridique implique deux choses. Premièrement, et alors que le droit romain connaît des distinctions essentielles entre les domaines (religieux, moral, juridique) et les pouvoirs (exécutif, législatif, juridique) d’abord, et entre le fait et le droit ou encore entre le droit et la loi ensuite, le droit nazi les annihile de son côté, privilégiant l'unification (ibid.: 208). Deuxièmement - et a fortiori -, ladite assimilation assure dans le même temps la création et l'application directe et immédiate du droit, puisque, précise le Commandant, il apparaît «tel qu’à la minute du jugement le juge en dernière analyse le conçoit» (Aragon 2000: 1225). En cela, le geste fondateur du juge relève d'une «décision qui n'appartient à aucun continuum temporel» (Ost 1999: 60, citant Ricœur), ce dernier étant affranchi d’une quelconque loi dans la mesure où "c'est lui qui doit la fonder comme à venir dans la violence» (ibid.), pour reprendre la thèse de Derrida (2005: 88-91). En l'espèce en effet, tant la genèse de la loi que son application se dotent d'une « dose non négligeable de violence et d'imposture " (Ost 1999: 6o), dès lors qu'elle dépend de la seule subjectivité de celui qui la fonde.

La démarche du Commandant rappelle celle entamée par quelques juristes allemands pour instituer ce nouveau droit. L'on pense notamment à Carl Schmitt pour qui le droit exprime avant tout un rapport de puissance, une idée plaidée dans un cycle de conférences prononcées à Berlin à l'occasion du premier anniversaire de l'accession d'Hitler au pouvoir en $1934^{10}$. Schmitt a dans cette perspective soutenu les décisions du dictateur dans son texte Le Führer protège [défend] le droit (Der Führer schützt das Recht), dans lequel il explique que: «Le Führer protège [défend] le droit contre le pire abus, lorsqu'au moment du danger, en vertu de ses fonctions de Führer et dans sa qualité de juge suprême, il crée du droit de manière directe et immédiate »" (Schmitt 1988: 20o, je souligne). À la lumière du conte d'Aragon, la formule - «il crée du droit de manière directe et immédiate" - est symptomatique. La "pulsion de pouvoir",

10 Les conférences sont publiées dans Les trois types de penséejuridique. Voir aussi à ce sujet Loi et jugement. Une enquête sur le problème de la pratique du droit (Gesetz und Urteil. Eine Untersuchung zum Problem der Rechtspraxis, 1912), un ouvrage de Carl Schmitt récemment présenté et traduit par Rainer Maria Kiesow, qui insiste d'ailleurs en introduction sur l'intérêt du couple «loi et jugement ", dès lors qu'« il n'y a pas de meilleure juxtaposition que la mise en opposition radicale de ces deux concepts" pour "comprendre quelque chose du monde juridique" (Schmitt 2019: 11).

11 «Der Führer schützt das Recht vor dem schlimmsten Missbrauch, wenn er im Augenblick der Gefahr kraft seines Führertums als oberster Gerichtsherr unmittelbar Recht schafft.» 
érigée au rang de "pulsion originaire" par Derrida (2000: 47-48), s’impose dans la locution performative du seul «je peux». La législation allemande ne s'autorise que d'elle-même, car, «armée de sa seule violence fondatrice, produit d'un acte performatif pur [...], suspendue dans le vide du non-droit, cette prétention n'aurait de comptes à rendre à personne " : le coup de droit est un coup de force (Ost 1999: 60).

Ce n'est pas au sens métaphorique mais c'est au sens littéral que Kafka exploite quant à lui l’image de la machine dans La colonie pénitentiaire. «C'est un appareil spécial» (Kafka 2018: 121), annonce d'abord fièrement l'officier au visiteur, car le système mécanique a dorénavant remplacé le labeur manuel ; “[il] va fonctionner tout seul» (ibid.: 122), spécifie-t-il ensuite. La description du mécanisme de la machine occupe toute la première partie de la nouvelle ${ }^{12}$, l'officier lui conférant une place centrale au sein du récit, dont il faut ici restituer les détails significatifs. Le condamné est allongé nu et à plat ventre sur une pièce appelée le lit, recouverte de ouate. Ses mains, ses pieds et son cou sont attachés à des courroies. Afin d'empêcher le condamné de crier ou de se mordre la langue, un petit bourrelet de feutre est coincé dans sa bouche - un tampon par ailleurs inchangé depuis une centaine de condamnés -, installation astucieuse puisqu'elle permet également d'éviter que la courroie du cou brise la nuque du malheureux. Une fois la machine enclenchée, le lit se met alors à vibrer en saccades rapprochées, de sorte à le faire glisser de droite à gauche et de haut en bas.

Lors de la présentation, un intérêt tout particulier est concédéà une pièce maîtresse de la machine, la herse, puisque le mécanisme global est rigoureusement agencé à celle-ci, dont la forme correspond à celle de l'être humain. Composée d'aiguilles, c'est elle qui a pour but l'exécution proprement dite du jugement. «Et c'est alors que le spectacle commence.» (Ibid.: 129) Les dents de la herse enfoncent leurs pointes dans la chair et inscrivent sur le corps du condamné sa sentence; les grandes gravent les lettres, alors que les petites, accolées, projettent de l'eau afin de laver le sang et de "faire en sorte que l'inscription demeure toujours lisible» (ibid.). Les aiguilles ne progressent pas de manière aléatoire, mais suivent les croquis calligraphiés par l'ancien Commandant, des tracés labyrinthiques qui se chevauchent. L'écriture ne saurait être trop simple, car elle ne doit pas tuer tout de suite, pas avant douze heures. Au fil de la manœuvre, le condamné est tourné pour offrir à la herse des espaces vierges et, entre-temps, les endroits écorchés par le graphe sont épongés par la ouate. Des petites dents sur la herse arrachent en outre les fils de coton éventuellement coincés dans

12 Principalement les pages 124-132. La description qui suit emprunte à celle de la nouvelle. 
les blessures pour préparer le corps aux nouvelles inscriptions, en palimpseste, plus profondes cette fois. Le texte se termine sur le visage, de sorte que le condamné puisse déchiffrer le texte en pointant la langue.

\section{Le rapport à la langue}

Force est alors de constater que les deux machines partagent un point commun dans leur rapport singulier au langage: elles donnent à voir le pouvoir des mots. Dénué de codification, le droit germanique décrit dans le texte d'Aragon - cette «machine de guerre» - n'est pour autant pas dénué de tout langage. Le juge envisage en effet son

118 devoir de juriste comme consistant à «élaborer un vocabulaire juridique, permettant aux seuls Allemands d'appliquer les règles favorables à [la] patrie, qui, le cas échéant, pourraient se retourner contre elles " (Aragon 2000: 1225). Et de préciser : “C'est là notre tâche, à nous, juristes de la vieille école, ralliés aux idées nouvelles. » (Ibid.) Contrairement aux gens de la Gestapo et aux «jouvenceaux de la police d'État» qui ignorent le "pouvoir des mots", le Führer l'a toujours considéré, précise encore le Commandant, car il sait «la nécessité de les détourner au profit de la cause allemande " (ibid. $)^{13}$. Autrement dit, l'institution du droit allemand - et son application - relève de l'élaboration d'une langue accessible aux seuls Allemands ${ }^{14}$, interdisant de fait sa compréhension aux condamnés.

La démarche institutionnelle ne va pas sans rappeler celle de La colonie pénitentiaire, qui atteint un paroxysme sous l'égide de la machine à la herse. D’abord, les graphes inscrits en sentence sur le corps du condamné ne sont lisibles que pour le bourreau, le visiteur, invité à plusieurs reprises à lire les croquis de l'ancien Commandant, échouant sans cesse à les déchiffrer. Ensuite, l'officier élude les interrogations du voyageur lorsqu'il s'intéresse de trop près aux déroulements de la procédure, répétant un simple «non": non, le condamné ne comprend pas la langue française, non, il n’a dès lors pas pu se défendre, non, enfin, il ne connaît pas sa peine; ce serait par ailleurs inutile, puisqu'il l'apprendra sur son propre corps, explique l'officier (Kafka 2018: 126). Enfin, bien sûr, les termes du jugement sont gravés dans la chair du condamné, la machine représentant à cet égard «l'objectivation d'une forme d'exercice du pouvoir » (Lahire 2011: 50).

\footnotetext{
13 Babilas rappelle par ailleurs un thème largement exploité par Aragon, le pouvoir des mots et leur détournement sémantique, notamment dans «L'homme contre les nuages ", discours prononcé le 13 mai 1939 et publié dans Commune en juin de la même année (Babilas 2004: 207).

14 L'on peut sur cette question se référer à l'ouvrage Lingua Tertii Imperii (1947) du philosophe et philologue Victor Klemperer, dans lequel il examine les conséquences (il parle d’empoisonnement) de la langue développée et employée par les nazis durant le Troisième Reich sur la culture et l'identité allemandes.
} 
Rendre la justice implique pourtant que "tous les "sujets" concernés sont supposés compétents, c'est-à-dire capables d'entendre et d'interpréter; tous les "sujets", c'est-à-dire ceux qui établissent les lois, ceux qui jugent et ceux qui sont jugés ", écrit Derrida dans Force de loi (2005: 40). Il serait en revanche «injuste de juger quelqu'un qui ne comprend pas ses droits, ni la langue dans laquelle la loi est inscrite ou le jugement prononcé" (ibid.: 40-41), poursuit-il avant de rappeler : "Comme on le sait bien, dans de nombreux pays, dans le passé et aujourd'hui encore, une des violences fondatrices de la loi ou de l'imposition du droit étatique a consisté à imposer une langue à des minorités nationales ou ethniques regroupées par l'État. " (ibid.: 47) Si le phénomène pointé du doigt par Derrida est dénoncé dans la nouvelle de Kafka, l'écrivain le retranche encore dans ses extrêmes. En effet, alors qu'un texte comme Le procès (1925), par exemple, relatant l'errance de Joseph K. dans les bas-fonds des tribunaux, illustre parfaitement l'inaccessibilité au droit, La colonie pénitentiaire la déjoue. Non seulement la loi est lue au prisonnier, mais elle est, en sus, inscrite dans sa chair; autrement dit, même lisible - à tout le moins perceptible -, le droit mène à la mise à mort.

To enforce the law, rappelle Derrida au début de sa communication. Loin de n'être qu'une simple minauderie rhétorique, le philosophe glose au travers l'expression, qu'il juge irremplaçable en français, le rapport entre langage et violence. "Au commencement de la justice, il y aura eu le lógos, le langage ou la langue, mais cela n'est pas nécessairement contradictoire avec un autre incipit qui dirait: Au commencement il y aura eu la force" (Derrida 2005: 26), précise-t-il. La loi est la loi. La locution ne dit rien mais, pourtant, elle fait sens, car elle est porteuse d'une force, elle est plus encore l'expression même de cette force, précise Jérôme Lèbre dans Derrida : la justice sans condition (2013: 11). Dans cette perspective, le juge n'applique pas les lois parce qu'elles sont justes, ni parce qu'elles résultent d'une démarche rationnelle, mais bien parce qu'elles sont des lois. Gewalt, en allemand, témoigne d'ailleurs du côté obscur du droit, car le terme signifie à la fois « violence » et " pouvoir légitime, autorité, force publique» (Derrida 2005: 19). Par suite, s'il y a des lois non appliquées, il n'y a pas de loi sans applicabilité, et donc sans force. To enforce the law, c'est faire allusion à une force inhérente à la loi qui la légitime et l'assure: «Il n'y a pas de droit qui n’implique en lui-même, a priori, dans la structure analytique de son concept, la possibilité d'être "enforced", appliqué par la force.» (Ibid.: 18) Bien que la traduction française lui fasse perde de sa valeur - appliquer la loi -, l'expression anglaise traduit en revanche une puissance inhérente et essentielle qui, bien qu'injuste ou injustifiable parfois, «est toujours une force autorisée " (ibid.: 17). 


\section{Le rapport au corps}

La description du décor où se situent les machines participe à cet égard de l'expression d'une force et, partant, d'une violence. L'«imposant» appareil (Kafka 2018: 124) de La colonie pénitentiaire se trouve en effet dans un "vallon encaissé, sablonneux et isolé par une ceinture de pentes dénudées" (ibid.: 121). La vallée est par ailleurs "sans ombre, le soleil agissait avec bien trop d'intensité, on avait du mal à rassembler ses pensées " (ibid.: 123). Le tribunal allemand dans Le droit romain n'est plus est de son côté logé dans " une bâtisse immense, disproportionnée à tout, à ce qui s'y passe, au siècle, à la ville. [...] Une demeure noire et haute, qui fait autour d'elle les rues étroites plus qu'elles ne le sont [...]. Cela écrase tout ce qui l'entoure.» (Aragon 2000: 1222) La force - ou peutêtre davantage la faiblesse - du droit, est ici exprimée à travers la démesure du lieu, dont l'hypotypose privilégiée par l'auteur dans sa description met à jour les conséquences d'une condamnation insensée.

L'espace du tribunal est d'autant plus terrifiant qu'il autorise une parole performative. En raison de la force inhérente à la loi, il suffit d'un simple «je peux " pour rendre le jugement exécutoire. Dépourvue de sens, écrasant tout ce qui l'entoure, l'autorité constitue ainsi le seul juge. Autrement dit, le droit n'existe dans les deux textes qu'à travers un pouvoir qui s'exerce sur des vies nues: inaccessibilité, irrationalité, incompréhensibilité ${ }^{15}$. Cette hypothèse est notamment développée par le philosophe Giorgio Agamben dans Homo Sacer, dans lequel il soutient l'idée que le droit et la vie sont indissociables. Dans la perspective foucaldienne du bio-politique, Agamben part du principe que la vie nue (zôè), pour reprendre le sous-titre de son ouvrage, a progressivement coïncidé avec l'espace politique, la rendant consubstantielle de ce dernier et, par conséquent, dépendante. L'homme nu étant un homme sans droit, l'espace du camp durant la Seconde Guerre mondiale constitue l'exemple même d'un lieu où le droit est suspendu et dans lequel le corps devient la cible par excellence du pouvoir. Et la continuité, voire la contiguïté, entre le droit et la chair est bien au ccur même de la machine de La colonie pénitentiaire, puisque «les aiguilles dans[ent] sur la peau» (Kafka 2018: 147). À son corps défendant, le condamné fait l'expérience d’un jugement qui annihile la distinction entre les deux entités (vie et politique).

Moins frontale quoique plus désarmante peut-être, l'expérience du bio-politique ${ }^{16}$ se lit aussi dans le texte d'Aragon, à travers la musique des os meurtris, dont l'hymne

15 Cette thèse est notamment discutée par Rainer Maria Kiesow au fil des chapitres de L'unité du droit (2014a). 16 La thèse du bio-politique est formulée par Michel Foucault dans La volonté du savoir (1976). 
monstrueux rythme la narration. À maintes reprises, et à la manière d'un refrain, Lotte se plaint d'une absence de musique. «Fräulein Lotte Müller, un peu de musique ne vous ferait pas de mal... Fräulein Lotte Müller, êtes-vous sourde que vous n'entendez pas la musique?» (Aragon 2000: 1232) apostrophe alors le narrateur, révélant crescendo la nature véritable de la mélodie en question. Si celle-ci est ponctuellement convoquée pour décrire les actes de violence - des coups de feu devant l'Uniprix à l'explosion d'un train de munitions (ibid.: 1233, 1234) -, elle exprime plus généralement le «lamento sourd des prisons d'où s'élèvent les plaintes déchirées d'instruments inconnus qu'on appelait des hommes" (ibid.: 1233). D’abord discrète, elle exhale un simple murmure : «On ne fait encore qu'accorder les violons dans la fosse. » (Ibid.) ${ }^{17}$ Elle emplit toutefois rapidement

tout autour de la ville le vaste paysage immobile et muet, elle y tourne, elle y monte, elle s'y déverse, avec les vents subitement levés d'un printemps tardif [...]. Allez, allez, ce n'est qu'un petit prélude encore... le grand orchestre ailleurs exercé se rassemble et la musique, la musique va jaillir! (Aragon 200o: 1235)

Touchant à son paroxysme, elle fait finalement entendre «le bruit des os broyés, le grésillement noir des chairs, le concert des tortures, les cris de la douleur morale si différents de ceux de la douleur physique, la basse rythmée des coups, le chant du sang clair qui jaillit, les larmes, les larmes, les larmes...» (ibid.: 1233).

Dans les deux cas, l'application de la loi appelle donc simultanément l'exécution du jugement - to enforce the law -, puisque l'observation immédiate de celle-ci laisse dans le même temps ses stigmates sur la chair ou les os des condamnés, confirmant l'idée d'une coïncidence entre la vie et la politique, entre le droit et le pouvoir. La prononciation de la décision judiciaire implique par conséquent la consécration d'un acte de violence, instituant un rapport tautologique entre eux deux et confirmant l'adage de Derrida : il n'y a pas de droit sans force (Derrida 2005: 17-18). À noter encore en incise que le performatif, en l'espèce, est aussi performance, dans la mesure où la condamnation fait l'objet, dans les deux récits, d'un spectacle. Si peu de spectateurs assistent

17 "J'entends le violon préluder dans la fosse", écrivait déjà Aragon en 1941 dans le poème «La nuit d'exil». C'est de manière plus générale un leitmotiv de la poésie d'Aragon depuis «Pour un chant (national)" publié en 1942. Dans «L'année du chèvrefeuille », paru en 1943, Aragon, sous le pseudonyme de Georges Meyzargues, écrit: «soufflez, soufflez, malheurs de la nation! Vous faites gémir des violes abandonnées, et cette voix qui naît d'entre les cendres à une autre répond qui est le cristal d'un festin interrompu; le contrepoint se poursuit dans de lointains villages, et la romance où se croisent les thèmes de douleur est fait [sic] de tous les chants brisés." (cité par Seghers 1974: 232) 
désormais à la scène, le bourreau de La colonie pénitentiaire rappelle que l'exécution constituait du temps de l'ancien Commandant un divertissement populaire: «Toute la vallée grouillait déjà de monde; ils venaient tous rien que pour voir.» (Kafka 2018: 136) Quant à Lotte, elle précise que «le seul amusement décidément, c'est le tribunal» (Aragon 2000: 1233).

\section{Décider}

L'acte de juger

Trancher, c'est marquer une rupture entre ce qui aurait pu être et ce qui est, c'est sortir du champ du possible pour entrer dans une réalité imposée. Le terme s'apparente en cela à un couperet, l'acte de juger consistant à séparer, à dé-partager, comme le suggère le terme allemand Teil (Ricœur 1992: 22). Dans les deux récits, les lois sont à cet égard appliquées selon la disposition inaugurale de tous les Codes de procédure - le juge applique la loi -, afin de congédier l'incertitude et de mettre un terme au litige. L'adage est en outre exécuté de manière directe et immédiate par le Commandant et l'officier, amenuisant ainsi l'écart temporel - trop velléitaire - entre le préjudice et le jugement. Dans le tribunal de von Lüttwitz-Randau, les condamnations sont expédiées : "Nous avons décidé de partir après la séance du tribunal ", indique le juge peu avant le départ pour la campagne avec Lotte, «séance tout à fait banale. Deux condamnations à mort. » (Aragon 2000: 1236) Par ailleurs, l'accusation relatée dans La colonie pénitentiaire traduit encore cet empressement. Un capitaine a déposé une plainte le matin même au sujet de son domestique, mise par écrit par l'officier dans la foulée « et, juste à la suite, la sentence» (Kafka 2018: 128). Tout était simple. «Si j’avais commencé par convoquer l'homme et l'interroger, il n'en serait sorti que confusion " (ibid.), se défend l'officier ${ }^{18}$ - à la fois juge et bourreau : «C'était ni plus ni moins un meurtre.» (Ibid.: 149)

Suivre ou appliquer une règle de droit n'est toutefois jamais décider. C'est affecter, tout au plus. Un juge ne doit pas être "une machine à calculer», écrit Derrida dans Force de loi (2005: 51), le terme choisi étant par ailleurs révélateur, puisqu’il renvoie aux machines judiciaires dont il a été précédemment question. Si le droit est parfois présenté comme étant calculable - un juriste apprend à résoudre des cas -, il contient de manière intrinsèque une certaine incompréhensibilité. Alors qu'une simple opération mathématique ferait coïncider la résolution à la sentence, une décision

18 L'on pense à cet égard aux discussions qui occupent depuis quelques années les réflexions relatives au remplacement, à tout le moins pour les affaires mineures, des juges par des robots (en raison des avancées technologiques dans le domaine de l'Intelligence artificielle), puisque l'un des arguments, justement, est le gain de temps. 
dépasse quant à elle les frontières du verdict (ibid.: 53). Ainsi, " coup par coup, il faudra à chaque fois, décider, se prononcer, juger, et puis méditer si c'était ça, être juste " (Derrida 1985: 107, citant Lyotard).

Pour cette raison, Derrida accorde un crédit particulier au moment du jugement et sa pensée offre à cet égard des clés de lectures intéressantes. Dans sa conception, tout jugement implique une suspension temporaire du droit, sorte d'angle mort où une (dés)articulation entre la conservation de la règle d'une part et sa suspension d'autre part opère. Il précise à cet égard :

Pour qu'une décision soit juste et responsable, il faut que dans son moment propre, s’il y en a un, elle soit [...] conservatrice de la loi et assez destructrice ou suspensive de la loi pour devoir à chaque cas la réinventer, la re-justifier, la réinventer au moins dans la réaffirmation et la confirmation nouvelle et libre de son principe. (Derrida 2005: 51)

Lacte de juger distingue ainsi deux événements. D’un côté, la décision doit souffrir un temps d'indécidabilité, rompant définitivement avec tout principe de prévisibilité; de l'autre, elle doit, en raison même de cette incertitude, repenser la loi au cas par cas, une instabilité devant encore subsister dans la décision elle-même.

\section{L'indécidable}

«La faute ne fait jamais de doute» (Kafka 2018: 127), proclame l'officier dans La colonie pénitentiaire. Derrida doit s'en retourner dans sa tombe. Car pour décider, le juge doit au contraire faire l'expérience de l'aporie, au sens aristotélicien du terme, c'est-àdire faire l'expérience du doute, de l'impossible. Pour le dire autrement, une décision ne peut jamais être une fin, le résultat d'une logique ou d'un calcul, mais elle doit être un commencement. "On ne peut décider que de l'indécidable, puisque sinon la simple connaissance suffirait.» (Kiesow 2014a : 114) Par suite, la décision ne résulte pas de la stricte application d'une règle, mais elle relève d'abord de l'événement, conférant une importance à ce qui se passe dans l'acte du jugement. Dans cette optique, "une décision qui ne ferait pas l'épreuve de l'indécidable ne serait pas une décision libre, elle ne serait que l'application programmable ou le déroulement continu d'un processus calculable», explique Derrida, avant de préciser : «Elle serait peut-être légale, mais elle ne serait pas juste.» (Derrida 2005: 53) La distinction - légale/juste - est ici fondamentale. Si la légalité prescrit la seule connaissance - biaisée cependant dès lors que le juge ne connaît jamais toutes les lois -, la justice, en revanche, déconstruit d'emblée toute présomption à la certitude, toute illusion à l'évidence de la décision simplement parce 
qu'une loi a été appliquée. En refusant d'envisager le droit comme l'“ agencement mécanique de règles comme le voudrait le positivisme juridique " (Michel 2012 : 76), Derrida renverse par conséquent le principe central qu'est la sécurité du droit pour peut-être y voir autre chose, autrement.

Faut-il encore préciser la notion d'indécidable. Celle-ci n’exprime pas seulement "l'oscillation entre deux significations [...]. [Elle] n'est pas seulement l'oscillation ou la tension entre deux décisions. Indécidable est l'expérience de ce qui, étranger, hétérogène à l'ordre du calculable et de la règle, doit cependant - c'est de devoir qu'il faut parler - se livrer à la décision. » (Derrida 2005: 53) L'intérêt pour l'espace médian - inbetween, dazwischen (Kiesow 2014a : 109) - fait dès lors sérieusement dévier la rationalité et la cohérence du droit ; au cheminement rectiligne (avant/après), Derrida préfère le parcours oblique et nécessairement instable (pendant), dont la décision garde par ailleurs la trace. L'entre-deux, l'intermédiaire favorisent alors un autre itinéraire entre la loi et la décision, qui s'épanouit en mi, mi-épais, mi-transparent, mi-obscur, mi-clair : et c'est dans cet espace trouble et indécis que « se fait la rencontre, se produit la liaison de la loi et du jugement" (ibid.: 111).

Logé dans un gouffre que «ni l'intention du législateur ni la lettre du texte ne suffisent à combler» (Ost 2012: 81), "l'instant de la décision est [en cela] une folie» (Derrida 2005: 58, se référant à Kierkegaard). Parce que le juge ne délibère pas sur l'absolu mais sur le contingent, la décision procède d'un saut dans le vide dont aucune certitude ne peut garantir la chute. Nulle décision sans doute. L'application rationnelle de la loi devient alors caduque, car le juge poursuit une démarche irrationnelle, un constat amenant Derrida à redéfinir, aux termes de son argumentaire, la notion même de justice: "Infinie, incalculable, rebelle à la règle, étrangère à la symétrie, hétérogène et hétérotrope. " (ibid.: 48) Pour autant, «ce moment de suspens angoissant ouvre aussi l'intervalle de l'espacement où des transformations, voire des révolutions juridicopolitiques, ont lieu " (ibid.: 46), car l'espace du jugement, me semble-t-il, n'est pas pour autant un lieu vide et abyssal; il est au contraire un lieu plein qui demande seulement à être ébranlé, déplié.

\section{La réinvention de la loi}

Cette manière de considérer le jugement invite à réfléchir sur ses modalités, notamment sur son rapport à la loi. Pour être juste, la décision doit pour mémoire «non seulement suivre une règle de droit ou une loi générale mais elle doit [aussi] l'assumer, l'approuver, en confirmant la valeur, par un acte d’interprétation réinstaurateur, comme si à la 
limite la loi n'existait pas auparavant, comme si le juge l'inventait lui-même à chaque cas" (ibid.: 50-51, je souligne). Le «comme si » est important. Alors que le Commandant von Lüttwitz-Randau l'élague, fondant son jugement sur l'intérêt national «tel qu'à la minute du jugement le juge en dernière analyse le conçoit " (Aragon 2000: 1225), le juge selon Derrida ne peut ignorer les lois et les décisions préexistantes. Cependant, si le jugement peut certes répéter quelque chose ou se conformer à une loi antérieure, l'interprétation du juge, pour être libre, ne doit pas consister dans la seule conformité du jugement (Derrida 2005: 51). Par suite, "chaque cas est autre, chaque décision est différente et requiert une interprétation absolument unique, qu'aucune règle existante et codée ne peut ni ne doit absolument garantir» (ibid.), conclut Derrida, instituant ainsi un hiatus fondamental entre les deux conceptions du droit.

Si une décision ne peut se borner à appliquer la loi, elle doit alors la réinventer, la rejouer à chaque fois (Michel 2012 : 76), évitant le fossé entre le général et le particulier. Pour reprendre l'analogie proposée par François Ost, le juge n'est pas un Jupiter, qui prône un droit inflexible, fixé et figé par avance, mais bien davantage un Hercule, qui, à l'inverse, privilégie un droit qui s'ajuste au cas par cas: «C'est la décision et non la loi qui fait autorité.» (Ost 1991: 243) Ce n'est d'ailleurs pas un hasard que l'on parle "d'appréciation du juge», la loi étant construite à chaque procès, c'est là qu'on la fait parler. La question de l'indécidable relève à ce titre d'un paradoxe: pour exercer la justice, il faut être libre et responsable de son action, de son comportement, de sa pensée et de sa décision; cependant, cette décision doit, pour être considérée comme telle, "suivre une loi ou une prescription, une règle" (Derrida 2005: 50). La décision implique donc que le juge construise lui-même les normes, indépendamment des lois promulguées, tout en revendiquant, dans le même temps la (re)connaissance du droit (Kiesow 2014a: 100). Voilà une " tache aveugle " (ibid.: 101) cruciale du principe de décision, puisque celui-ci s'exerce à la fois hors et dans la loi. Hors la loi d'abord, parce que le verdict ne résulte pas d'une simple équation mathématique, mais d'une poïesis, d'une création continuelle de la loi, au cas par cas. Dans la loi ensuite, car la décision ne peut jamais se désintéresser tout à fait de la loi, puisqu'elle doit, afin d'assurer une certaine légitimité, reposer sur un principe de droit reconnu et sécurisant.

Cette dualité permet néanmoins de repenser le couple, biaisé, droit/loi, Derrida invitant d'emblée à les dissocier (Derrida 2005: 17). La loi n'est radicalement pas le droit. Elle désigne un ensemble de règles générales et abstraites érigé par un législateur, lui conférant avant tout une dimension politique. L'on obéit aux lois non parce qu'elles sont justes, mais parce qu'elles sont des lois, c'est le fondement mystique de 
leur autorité, pour emprunter le sous-titre de l'ouvrage de Derrida. Par conséquent, si l'on se plaît souvent à parler de «l'application de la loi» en matière de jugement, c'est créer une confusion pour éluder certains enjeux, dérangeants. Car l'acte de jugement n'a rien à voir avec la loi ; il a à voir avec le droit. Contrairement à la loi, il est un ensemble hétéroclite, sans consensus et instable. Cela dérange, bien sûr, car cela remet sérieusement en question la notion de "sécurité juridique » et d'«état de droit». "La déconstruction est la justice» (ibid.: 35), conclut à cet égard Derrida. La justice avec un grand J, celle qui n'est jamais figée. Si Derrida lui-même se refuse à donner une définition stricte à la déconstruction - ni tout à fait théorie ni tout à fait méthode -, il est cependant possible de la comprendre comme le levier qui permet d'ouvrir un questionnement. Son but n'est pas de détruire, dans une démarche nihiliste, mais davantage d'ébranler, de déstabiliser, voire de fissurer des locutions figées ou des concepts cristallisés, à l'instar du couple loi/droit. Cette manière de considérer l'acte de jugement révèle une zone d'ombre non négligeable dans les deux textes: le juge est certes lié à la loi, mais pas seulement, il est également lié au droit, qui lui cède alors une importante responsabilité.

\section{Se positionner}

\section{La responsabilité à l'épreuve}

"Il y a quelque chose de pourri au cœur du droit», retient Derrida de la Critique de la violence de Benjamin (ibid.: 94-95), faisant référence à une violence qui, hors la loi mais à l'intérieur du droit, exerce sa puissance. Si le contexte d'énonciation est celui de sa fondation et de son institution, cette célèbre phrase de Benjamin retentit avec peut-être davantage de force à la lumière des deux récits du corpus, plus spécifiquement avec les deux mises en scène du jugement - et de son exécution. Outre une application directe et immédiate de la loi en effet, c'est-à-dire sans faire l'expérience aporétique du doute, les textes dénoncent une application dirigée de celle-ci, amenuisant sérieusement l'écart entre une mise en œuvre mécanique et manuelle de la machine judiciaire : non seulement la décision est orientée, mais elle est encore assumée comme telle.

Lorsque le Commandant von Lüttwitz-Randau, au moment de son arrestation, clame pour sa défense «je n’ai jamais tué personne [...]. Je suis un magistrat qui applique les lois", il ne fait que renforcer l'illusion d'un système pénal neutralisé et banalisé. Car le juge, dans les deux nouvelles, n'est-il, pour reprendre la célèbre formule de Montesquieu, que «la bouche de la loi» (Montesquieu 1951: 404), comme le prétend le Commandant? Non. Le juge militaire allemand et l'officier n'ont pas seulement appliqué et suivi la loi, ils ne se sont pas seulement conformés au droit et 
aux institutions alors en vigueur, ils y ont pleinement adhéré, révélant ainsi quelque chose de plus inquiétant, de plus déroutant: l'entrée en jeu de l'individualité des décisionnaires. Les deux juges interprètent la loi avec leur opinion, leur idéologie et leur personnalité. Ce n'est pas le système, ce n'est pas le droit, mais c'est eux; voilà, peut-être, la véritable force du droit.

Ce n'est donc pas une machine qui applique la loi, mais ce sont bien les juges. Refusant un rejet de la responsabilité sur le seul système - «c'est la guerre... la guerre est une chose triste", "c'est une terrible chose, la guerre" (Aragon 2000: 1249), scande le Commandant allemand une fois pris entre les mains des maquisards -, les deux récits préfèrent mettre en évidence une responsabilité parfois éludée, la littérature ayant à cet égard l'avantage de montrer cette subjectivité, de mettre en situation, plus que la théorie du droit pure. Par suite, le droit est tributaire d'une décision, plus que d'une interprétation. "Le droit est ce que les juges en font " (Kiesow 2014a : 12), affirme à ce titre Kiesow. Dans cette optique, et au moment de décrire les différents sens de juger, Ricœur en précise la caractéristique suprême : «Nous avons atteint le sens fort du mot juger : non seulement opiner, estimer, tenir pour vrai, mais en dernier ressort prendre position.» (Ricœur 1992: 21, je souligne)

\section{L'individualité}

À l'aune des textes retenus, l'idée de prise de position est une variable susceptible de dévoiler une autre face du droit dans le contexte totalitaire, puisqu'elle a le mérite de creuser un écart significatif entre droit et loi. L'Oberleutnant Willi, officier de la Gestapo dans Le droit romain n'est plus, ne se contente pas d'affecter une mesure aux cas juridiques, il en fait «sa spécialité» : «sa spécialité, ce sont les Juifs. Il en trouve partout. On dirait qu’il les fait naître. Il les fait bien mourir aussi", explique Lotte (Aragon 2000: 1220). Quant au Commandant von Lüttwitz-Randau, il n’adhère pas seulement aux idéaux nazis, ce sont ces derniers qui correspondent à sa manière de voir les choses, une configuration inversée qui gagne à être ici détaillée.

Lors du voyage en train d'abord, rêvant dans la moiteur du wagon, le Commandant allemand considère avec sympathie l'audace du Führer:

Le droit romain comme base des lois modernes, c'est une absurdité révoltante et contraire à l'esprit allemand. [...] Le Führer a été tout à fait inspiré d'anéantir toutes les lois, ce qui permet de rebâtir dans des conditions vraiment allemandes un droit qui n'a pas besoin de code. (Ibid.: 1237) 
Aux yeux du juge, la seule loi valable est donc celle pensée et instituée dans le seul intérêt de l'Allemagne, une idée par ailleurs largement répandue en Allemagne nazie, ainsi qu'en témoigne la formule «Oberstes Gesetz ist das Wohl des deutschen Volkes" ("La loi suprême est le salut du peuple allemand", ibid.: 1241). Conscient des enjeux d'une telle réédification du droit, il se sent alors personnellement investi d'une mission, un rôle qu'il justifie aux maquisards lors de son arrestation:

Je suis entré dans la ${ }^{19}$ parti, au lendemain de l'exécution de Röhm et de ses complices, [...] parce quej'ai alors tout de suite compris qu'il fallait des juristes pour, à la lumière de ce fait d'une importance historique... wie sagt man? réviser entièrement, wiederaufbauen... réédifier le droit allemand. (Ibid.: 1240)

Le choix des mots est alors révélateur d'un positionnement, en témoigne encore l'argumentaire relatif à l'affaire Dimitrov, accusé à tort d'avoir incendié le Reichstag avant d'être innocenté: «c'est justement, dit Lüttwitz-Randau, en ce temps-là nos tribunaux étaient encore infectés par le droit romain, le Code Napoléon, les lois juives... Aujourd'hui, jamais nous n’aurions laissé repartir Dimitrov, il aurait été condamné... selon le droit allemand " (ibid.: 1241, je souligne) - pour ne pas dire selon moi.

Ensuite, le Commandant insiste à plusieurs reprises sur l'affinité entre ses propres conceptions du droit et celles revendiquées, par la suite, par le Führer. «J'ai lu un article extrêmement intéressant dans le Völkischer Beobachter ${ }^{20}$ sur l'évolution du droit allemand", dit-il en rejouant sa conversation avec l'Oberleutnant Willi, "c'est très singulier, j'y ai retrouvé plusieurs vues que j'avais moi-même en 1925, huit ans avant la prise par notre Führer du pouvoir, dans ma thèse De jure gremanico, hasardées " (ibid.: 1224). Et d'en conclure: « ce qui prouve quej'ai beau n'être qu'un rallié, il n'y a pas moins entre le national-socialisme et moi de très anciennes et profondément troublantes affinités ", précise-t-il fièrement à son interlocuteur qui, lui, ricane (ibid.: 1224-1225). Ladéquation entre ses idées personnelles, exprimées dans sa thèse, et les idéologies nazies répandues par le Parti national-socialiste des travailleurs allemands (NSDAP) se concrétise alors dans le discours qu'il défend dans la seconde partie de la nouvelle, du voyage en train à son arrestation. «J'ai été professeur de droit romain. Mais, pour faire primer le droit germanique, il faut, c'est mon point de vue, effacer dans le monde moderne toute trace du droit romain " (ibid.: 1237), soutient-il, un point de vue amenant

19 Aragon a volontairement reproduit le français approximatif du Commandant. 20 L'Observateur populaire, organe de presse officiel entre 1920 et 1945. 
les maquisards à personnaliser le droit, puisqu'ils reprochent au juge d'avoir violé les lois selon "votre» droit (ibid.: 1241). Par conséquent, et malgré ce qu'en pense Lotte «ces familles aristocratiques n'arrivent pas à comprendre notre socialisme. Ni notre Führer" (ibid.: 1221) -, non seulement le juge le comprend très bien, mais, bien plus encore, il se l'approprie selon sa propre perception du droit (Babilas 2004: 201).

La fierté du juge relative à ses conceptions doctrinales est également un leitmotiv de La colonie pénitentiaire, puisque le clivage idéologique entre l'officier et le nouveau Commandant tisse le nœud de la nouvelle. Le premier est en effet le seul partisan de la méthode d'exécution au sein de la colonie, alors que le second défend une vision divergente de la justice. Si le texte ne la précise pas, la configuration binaire des deux protagonistes suggère une vision plus clémente, à tout le moins fermement opposée à l'usage de la machine à la herse. En outre, alors que le spectacle d'exécution suscitait jadis la clameur du peuple, la scène est aujourd'hui désertée par ce dernier, qui ne consent plus à sa procédure.

La sentence du texte - la machine sert à « rendre la justice» (Kafka 2018: 134), c'est à elle qu'est confiée l'exécution du jugement - est à cet égard un leurre. Bien que la machine fasse l'objet d'une critique de la chosification et de la réification du droit, elle est aussi au centre d'une réflexion sur la personne qui, derrière, l'actionne et la fait fonctionner. De manière chronique, le narrateur insiste sur le sentiment de satisfaction qui emplit l'officier à l'égard de son appareil. «En uniforme avec sa veste d'apparat collée au corps, alourdie par des épaulettes et bardée de fourragères " (ibid.: 123), ce dernier fait l'éloge du mécanisme au visiteur. Plus encore, il se plaît à signaler sa participation à son édification - de manière analogue, d'ailleurs, à l'engagement du Commandant von Lüttwitz-Randau pour réédifier le droit allemand: «Cet appareil [...] est une invention de notre précédent commandant. J'ai d'emblée collaboré aux tout premiers essais et j'ai pris part également à tous les travaux jusqu'à leur achèvement. " (ibid.: 122-123) L'implication fondatrice de l'officier sur laquelle il insiste lui sert par ailleurs de passe-droit à la fonction de juge. Il précise qu'il endosse ce titre parce qu'il connaît bien la machine: «l'affaire se présente de la manière suivante. Je suis affecté aux fonctions de juge dans cette colonie pénitentiaire. Malgré mon jeune âge. Cela parce que je secondais l'ancien commandant dans toutes les affaires pénales et parce que c'est moi qui connais le mieux l'appareil" (ibid.: 127), explique-t-il au visiteur, et ce bien que personne d'autre que lui n’adhère au principe (« la faute ne fait jamais de doute») qui le guide afin de trancher, pour prendre les mots du texte. 


\section{Le nœud de l'intrigue}

La dénonciation de l'individualité des deux personnages par les nouvelles du corpus est d'autant plus révélatrice que c'est bien la conception personnelle des deux juges qui est à l'origine de leur perte. Craignant que le visiteur, avec ses opinions européennes, n'entrave son travail en assurant la coalition du nouveau Commandant et en éradiquant sa pratique barbare, l'officier de La colonie pénitentiaire se place de son plein gré sous la herse, préférant mourir sous le joug de la machine plutôt que de vivre sans que son droit ne soit appliqué.

Le principe est d'autant plus symptomatique avec le Commandant von LüttwitzRandau, puisque le retournement de situation intervient après plusieurs tentatives des maquisards d'offrir une seconde chance au juge allemand, une abnégation de la part de ce dernier à laquelle participe en outre la description esthétisante du paysage, dont la force ne parvient à le faire fléchir. Emmené sur les lieux du crime, l’on fait visiter au Commandant le décor des supplices de «son » droit. Les maisons ne s'apparentent plus qu'à des dentelles, trouées, entourées d'un amoncellement de gravats, de fer rouillé, de terre remuée et de poutres écroulées où des filles ont été violées, des jeunes garçons fusillés et des yeux arrachés (Aragon 2000: 1247). Le paysage, palimpseste des luttes passées et acteur du présent drame, se fait alors de plus en plus menaçant ${ }^{21}$ :

Les crêtes dansent. Des montagnes sortent des montagnes comme les pigeons d'un chapeau. La lune si claire surtout que tout semble un danger mortel. [...] La lune, les pierres, la lune, le défilement noir des crêtes, à un tournant soudain blanchies, comme si le paysage virait au négatif. (ibid.: 1246)

Pour autant, le juge ne laisse paraître aucun remords, répétant seulement «une vieille phrase qui lui avait [...] servi en 1940-1942 [et qui] lui revint seule aux lèvres comme un hoquet: Wir sind doch keine Barbaren... " (ibid.: 1245).

À cet égard, si les actes d'accusation sont clairs, la défense assurée par le Commandant lui-même dévoile quant à elle un fonctionnement plus complexe (Babilas 2004: 209), ou, plus encore, dérangeant. Le narrateur lui soumet en effet deux

21 L'idée est notamment soutenue par Daniel Bougnoux: «Le paysage revêt un visage à la fois historique et mythologique, quand il devient dans "Le droit romain n'est plus" l'un des acteurs du drame, et que par le biais des maquis la nature entre dans l'Histoire. Cet élargissement de la scène ou de la conspiration, au fil de ces récits, suggère constamment l'organisation secrète sous la contrebande des apparences, une orchestration qui prélude en sourdine mais frémit, et se trouve sur le point d'éclater. " (Bougnoux 200o: 1440) 
issues possibles, deux lignes de défense à choix, mais le Commandant se tourne vers celle qui signe sa mise à mort. Il se défend en considérant «l'obéissance et la fidélité à son Führer comme la justification de tous ses actes, comme le point de droit qui le soustrayait à l'examen de toute justice " (Aragon 2000: 1242). Autrement dit, le juge en appelle à son "attitude intérieure" (Babilas 2004: 210), ou plus précisément à sa conviction personnelle. Si le choix de la confession, relative à la relation intime qu'il entretenait avec le Führer, sert de ligne de défense au Commandant, elle traduit plus généralement une variable non négligeable inhérente à l'acte de juger : l'individualité de celui qui prend la décision.

L'autre ligne de défense aurait consisté à dénoncer le système nazi et à se désolidariser du Führer, puisque l'obéissance du Commandant est considérée par les maquisards "comme une aggravation de cas, comme une preuve de culpabilité" (Aragon 2000: 1242). Un mensonge aurait suffi. Les maquisards, au fond, «lui donnaient sa chance en lui permettant de dire que tout cela, tout ce qu'on pouvait reprocher non pas seulement à lui, commandant von Machin Chouette, mais aux Allemands, tous les Allemands, c'était en fait leur Führer " (ibid.), une ligne de défense retenue par de nombreux accusés, concrétisant la banalisation du mal étudiée par Hannah Arendt notamment ${ }^{22}$. Un mensonge aurait cependant trahi le sentiment d'attachement profond qui lie le juge au droit allemand. Et au narrateur de conclure: «Il ne pouvait pas comprendre qu'il aurait sauvé sa peau en désavouant le national-socialisme. [...] en réalité c'était luimême, c'étaient ses propres paroles qui le condamnaient, qui le perdaient. Sa défense. " (Ibid.) Le pronom retenu - sa défense - confirme alors la corrélation entre la pensée du Commandant et celle du régime nazi. À jouer «à la grandeur, à la fidélité nationalsocialiste", le Commandant est ainsi "pris au piège de son propre système " (ibid.). Ce sont ses propres paroles, ses propres convictions qui le condamnent et amènent ses bourreaux au constat suivant: il est «inguérissable» (ibid.).

Les deux textes réalisent par conséquent un retournement de situation significatif, car, en jugeant les juges, ils opèrent un véritable renversement de perspective. Non seulement le juge militaire allemand du Droit romain n'est plus et le bourreau de La colonie pénitentiaire sont mis sur le banc des accusés, autrement dit sont déplacés de l'autre côté de la machine judiciaire, mais leur individualité, en outre, qui constituait en dernière analyse le critère ultime de leur prise de décision, se retourne brutalement

22 Pour reprendre le titre de l'ouvrage d'Hannah Arendt (1963), Eichmann à Jérusalem: rapport sur la banalité du mal. 
contre eux. Partant, c'est bien la confiance aveugle dans leur perception du droit qui finit par les faire condamner.

\section{Derrière le droit}

Les nouvelles de Kafka et d'Aragon ont ainsi le mérite de mettre en évidence certaines caractéristiques complexes du droit, dont la distinction avec la loi est centrale pour en cerner les contours. Les possibilités de la narration déplient en effet les strates du réel pour détourner et repenser quelques lieux communs - le juge applique la loi -, afin de révéler la tache aveugle inhérente à l'acte de juger, notamment en regard à la responsabilité de celui qui tranche. Répondre à une exigence de justice, selon Derrida, revient à répondre de ses paroles ou de ses actes (Lèbre 2013: 81). Cependant, "la responsabilité ne s'arrête jamais là ", car lorsqu'on "répond “de”, on répond aussi “devant": “devant un autre, une communauté d'autres, une institution, un tribunal, une loi". La responsabilité est [en cela] foncièrement en excès vis-à-vis de soi, elle en demande plus.» (Ibid.) À ce titre, les deux textes rendent compte des enjeux du jugement, en interrogeant ses modalités et ses limites, auxquelles l'approche de Derrida offre des pistes de réflexion utiles, dès lors qu'elle met en branle la machine judiciaire.

D’abord, les deux récits invitent à observer la violence constitutive de la structure même du droit, puisque sa fondation traduit l'expression d'une force, l'abolition du Code Napoléon au profit d'un droit «tel qu'à la minute du jugement le juge en dernière analyse le conçoit» (Aragon 2000: 1225) préconisée par le juge allemand dans Le droit romain n'est plus étant à cet égard symptomatique. Plus généralement, la thèse rejoint celle défendue par Robert Cover dans Violence and the World (1986), un ouvrage majeur dans lequel l'auteur considère que le droit, outre une attribution de sens, peut aussi constituer une justification d'actes de violence - un pan du droit d'ailleurs reproché par les maquisards au Commandant lors de son arrestation. Cela implique alors de rompre avec une vision intuitive de ce dernier, qui tend à neutraliser le système pénal par un vocabulaire stérilisé: système, rationalité, application. Derrida écrit d’ailleurs dans Force de loi, au sujet du jugement: "Ne jamais céder sur ce point, tenir constamment en haleine un questionnement sur l'origine [...], c'est, du point de vue d'une déconstruction rigoureuse, tout sauf une neutralisation." (Derrida 2005: 45) Les deux récits montrent qu'un point de vue neutralisé est biaisé, car la décision du juge est avant tout un acte situé qui vise quelqu'un, qui frappe et qui fracture le monde. Autrement dit, ils attirent l'attention moins sur ce que le juge dit que sur ce qu'il fait; et, objectivement, il inflige une violence, en témoigne, de façon aussi littérale que métaphorique, les aiguilles de la machine à la herse actionnée par l'officier dans La colonie pénitentiaire. 
Ensuite, et a fortiori, les deux nouvelles mettent le doigt sur une variable centrale du droit: l'humain. Trancher, décider dirait Derrida, implique une responsabilité que le calcul, par sa stricte application, interdit. Si le Commandant von Lüttwitz-Randau est condamné, ce n'est pas pour avoir effectué un jugement en raison d'une disposition juridique, aussi injuste soit-elle, c'est pour avoir délibérément réalisé ses propres convictions idéologiques. De même, l’officier dans La colonie pénitentiaire est guidé par son fanatisme pour la machine à la herse, alors même que l'autorité judiciaire, représentée par le nouveau Commandant, réprouve la pratique. Par conséquent, si la machine est placée au cœur des deux textes pour dénoncer la réification du système judiciaire, elle sert aussi à en dévoiler la face cachée. Derrière la machine, il y a l'humain. Cette variable conduit notamment Derrida à longuement gloser l'acte de juger, en accordant un intérêt particulier à la décision, distincte en cela de l'application (mécanique).

Rendre justice, d'une manière responsable, excède toujours le simple devoir de restituer ou de faire droit : c'est ne jamais compter sur la symétrie et l'équilibre d'un donné et d'un rendu, car cette logique du même suppose toujours que la même force, la même violence, soit exercée de part et d'autre. (Lèbre 2013: 82)

Partant, lorsqu'il décide, le juge réinvente - illusoirement du moins - la loi, rompant définitivement avec l'idée d'un droit unifié.

Dans cette perspective, l'on pense de manière décisionnelle et non plus causale, replaçant au centre de la réflexion l'humain et sa sensibilité. Cela invite à reconsidérer le droit, puisque celui-ci n'est plus envisagé comme normatif, stable et figé, mais qu'il est au contraire plastique, instable. Concevoir le droit comme une structure fixe rassure et sert certes de méthode à penser, mais cette structure ne peut le définir exclusivement. "Le droit n'est pas une pure théorie, mais une force vive», écrivait à cet égard Rudolf von Jhering dans La lutte pour le droit (2006): il n'y a peut-être rien derrière lui, si ce n'est nous-mêmes. 


\section{Références}

ARAGON Louis (2000 [1945]), «Le droit romain n'est plus ", in CEuvres romanesques complètes II, Paris, Gallimard, pp. 1219-1253

ArAgon Louis (2010 [1944]), "Épilogue d'un drame ", La Drôme en arme, $\mathrm{n}^{\circ}$ 4, facsimilé reproduit dans Les annales de la Société des amis de Louis Aragon et Elsa Triolet, $\mathrm{n}^{\circ} 12$, pp. 137-138.

Aхт Dieter (2017), «Entretien avec François Ost. Droit et littérature: les deux faces du miroir ", Revista Internacional de Direito e literatura, $\mathrm{n}^{\circ}$ 1, pp. 249-264.

BABILAs Wolfgang (2004), “"Le droit romain n'est plus”. Observations, remarques,

134 questions ", Les annales de la Société des amis de Louis Aragon et Elsa Triolet, $\mathrm{n}^{\circ}$ 6, pp. 193-216.

BAREÏT Nicolas (2016), “Lire Kafka après Foucault: In der Strafkolonie», Revue de science criminelle et de droit pénal comparé, $\mathrm{n}^{\circ}$ 2, pp. 255-261.

Bidim A Jean-Godefroy (2002), «L'acte de juger et le magistrat: de la précompréhension à l'occasion ", Rue Descartes, $\mathrm{n}^{\circ}$ 36, pp. 181-200.

Bougnoux Daniel (200o), "Servitude et grandeur des Français. Notice du recueil», in CEuvres romanesques complètes II, L. Aragon, Paris, Gallimard, pp. 1438-1440.

Bougnoux Daniel (2008), “"Le droit romain n'est plus”. Note sur un conte écrit par Aragon en 1944", Histoire de la justice, $\mathrm{n}^{\circ}$ 18, pp. 201-203.

DERRIDA Jacques (1985), «Préjugés : devant la loi », in La facultéde juger, J. Derrida et al., Paris, Minuit, pp. 87-139.

DERRIDA Jacques (2000), États d'âme de la psychanalyse. Adresse aux États Généraux de la Psychanalyse, Paris, Galilée.

DERRIDA Jacques (2005 [1994]), Force de loi. Le «Fondement mystique de l'autorité», Paris, Galilée.

JHERING Rudolf von (2006 [1872]), La lutte pour le droit, trad. par O. de Meulenaere, Paris, Dalloz.

KA F K Aranz (2018 [1919]), “Dans la colonie pénitentiaire», in Nouvelles et récits. Euvres complètes I, trad. par I. Kalinowski et al., Paris, Gallimard.

Kiesow Rainer Maria (2014a), L’unité du droit, Paris, EHESS.

KIESOW Rainer Maria (2014b), «La science à la barre », Socio, nº 3, pp. 199-208.

LAHIRE Bernard (2011), "Kafka et le travail de la domination", Actuel Marx, n 49, pp. 46-59.

LÈ BRE Jérôme (2013), Derrida : la justice sans condition, Paris, Michalon.

LeVel Maurice (1908), L'épouvante, Paris, Le Monde illustré. 
Michel Johann (2012), Ricceur et ses contemporains: Bourdieu, Derrida, Deleuze, Foucault, Castoriadis, Paris, Presses universitaires de France.

MONTeSQUieu (1951 [1748]), “De l'esprit des lois », in Euvres complètes, Paris, Gallimard, pp. 227-995.

Ost François (1991), "Jupiter, Hercule, Hermès: trois modèles de juges ", in La force du droit. Panorama des débats contemporains, P. Bouretz (dir.), Paris, Esprit, pp. 241-272.

Ost François (1999), Le temps du droit, Paris, Odile Jacob.

Ost François (2012 [2007]), Dire le droit, faire justice, Bruxelles, Bruylant.

Ost François (2015), «Droit et littérature: variété d'un champ, fécondité d'une approche ", Revue juridique Thémis de l'Université de Montréal (RJTUM), nº 1, pp. 3-33.

RICÆU R Paul (1992), “L'acte de juger », Esprit, n 183, pp. 20-25.

Sснмітт Carl (1988 [1934]), "Der Führer schützt das Recht», Positionen und Begriffe, Berlin, Duncker \& Humblot.

SCHмitт Carl (2019 [1912]), Loi et jugement. Une enquête sur le problème de la pratique du droit, trad. par R. M. Kiesow, Paris, EHESS.

SEgHeRs Pierre (1974), La Résistance et ses poètes. France 1940-1945, Paris, Seghers. 